\title{
Detection of CdS Nanoparticles and Implications for Cadmium Yellow Paint Degradation in Edvard Munch's The Scream (c. 1910, Munch Museum)
}

\author{
Barnaby D.A. Levin ${ }^{1}$, Kayla X. Nguyen ${ }^{1}$, Megan E. Holtz ${ }^{1}$, Marcie B. Wiggins ${ }^{2}$, Malcolm G. \\ Thomas $^{3}$, Eva S. Tveit ${ }^{4}$, Jennifer L. Mass ${ }^{5}$, Robert Opila ${ }^{6}$, Thomas Beebe ${ }^{2}$, David A. Muller ${ }^{1,7}$. \\ 1. School of Applied and Engineering Physics, Cornell University, Ithaca, NY, USA. \\ 2. Department of Chemistry and Biochemistry \& UD Surface Analysis Facility, University of \\ Delaware, Newark, DE, USA. \\ 3. Cornell Center for Materials Research, Cornell University, Ithaca, NY, USA. \\ 4. The Munch Museum, Tøyen, Oslo, Norway. \\ 5. Department of Conservation, Rijksmuseum, Amsterdam, NL. \\ 6. Department of Materials Science and Engineering, University of Delaware, Newark, DE, USA. \\ 7. Kavli Institute for Nanoscale Science, Cornell University, Ithaca, NY, USA.
}

Cadmium sulfide (CdS) based yellow paint is fading, flaking, and discoloring with age in billions of dollars worth of Impressionist through Expressionist masterpieces from the late $19^{\text {th }}$ and early $20^{\text {th }}$ centuries. Characterization of the morphology, chemistry, and crystal structure of paint particles is critical for understanding CdS pigment degradation, and the role of other cadmium compounds in paint synthesis and aging [1]. Here, we use scanning transmission electron microscopy (STEM) to identify nanoparticle structures in a sample of cadmium yellow paint from the Edvard Munch's The Scream (c. 1910, Munch Museum), taken from a region of flaking yellow paint in the water adjacent to the two background figures on the bridge (Fig. 1a), and prepared for STEM by focused ion beam (FIB) milling.

Spectroscopic mapping of the paint sample by electron energy loss spectroscopy (EELS) shows that although most of the particles in the sample are cadmium compounds (Fig. 1b), only a small minority contain sulfur (Figure 1c), suggesting that much of the CdS that would have originally been present at synthesis has degraded. EELS mapping reveals that a majority of paint particles in the sample consist mainly of cadmium carbonate $\left(\mathrm{CdCO}_{3}\right)$. Compounds such as $\mathrm{CdO}, \mathrm{CdCl}_{2}$, and $\mathrm{CdCl}(\mathrm{OH})$ may also be present, as observed in XEDS maps of a nearby FIB section, which showed similar carbonate/sulfide distributions, and in $\mu$ XANES analysis of paint from the same region.

Crystallographic datasets with a sub-nm probe were acquired using a high-speed pixel array detector [2]. Large $(>10 \mathrm{~nm})$ crystal grains in the sample were mapped using the asymmetry of diffraction patterns from datasets acquired at different sample tilts. Overlaying the crystal grain map and sulfur EELS signal shows that almost all CdS particles are located in regions with no large crystal grains (Fig. 2a). The diffraction patterns from CdS particles show a symmetric polycrystalline ring shape, indexed to cubic Hawleyite (Fig. 2b). From probe size and sample thickness, this suggests that the CdS consists of small (<10 nm) nanocrystalline particles; closer examination by ADF STEM (Fig. 2c) shows CdS nanoparticles in the range of 2-10 nm. CdS is highly photosensitive to oxidation [3], and $\mathrm{CdS}$ nanoparticles are particularly reactive due to their high surface area to volume ratio. This may be a key reason for the aging of cadmium yellow paint in the Scream. Given the higher reactivity of smaller nanoparticles, we hypothesize that smaller $(\sim 10 \mathrm{~nm})$ and rounded $\mathrm{CdCO}_{3}$ grains are $\mathrm{CdS}$ aging products, while larger $(\sim 100 \mathrm{~nm})$, well-faceted $\mathrm{CdCO}_{3}$ crystal grains were present from paint synthesis [4]. 
[1] E. Pouyet et al, Appl. Phys. A 121, (2015) p.967.

[2] M.W. Tate. et al, Microsc. Microanal. 22, (2016) p.237.

[3] Hsieh \& Huang, Colloids and Surfaces, 53 (1991) p. 275.

[4] Electron Microscope facility support from NSF MRSEC program (DMR-1120296).

a)
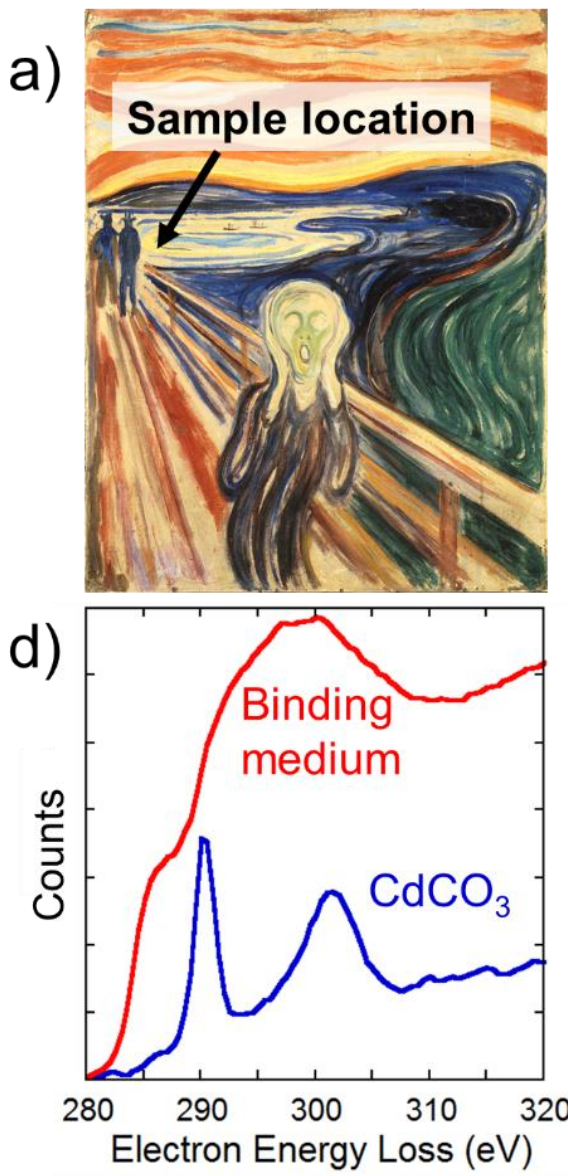

b)

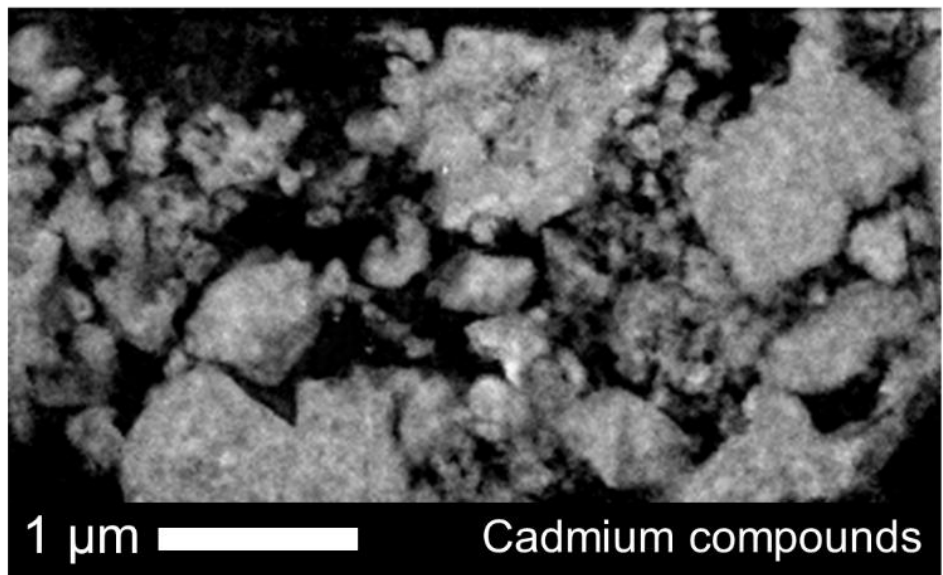

c)

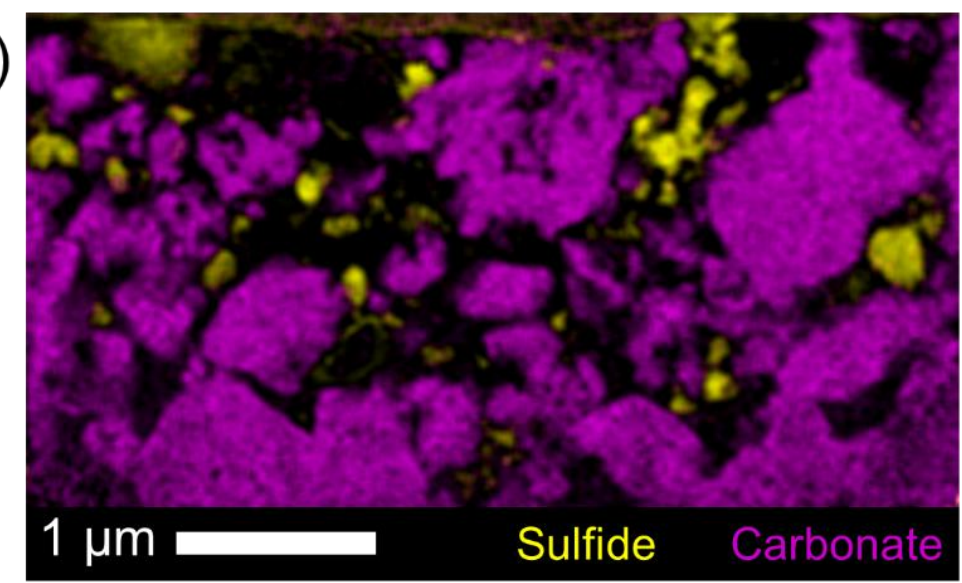

Figure 1. a) Approximate paint sampling location on "The Scream" indicated by arrow (Photo () Munch Museum). b) EELS map showing cadmium compound particles present throughout paint layer. c) Composite EELS map showing cadmium carbonate and cadmium sulfide particles d) Carbon Kedge EELS spectra of carbonate, and organic binding medium.
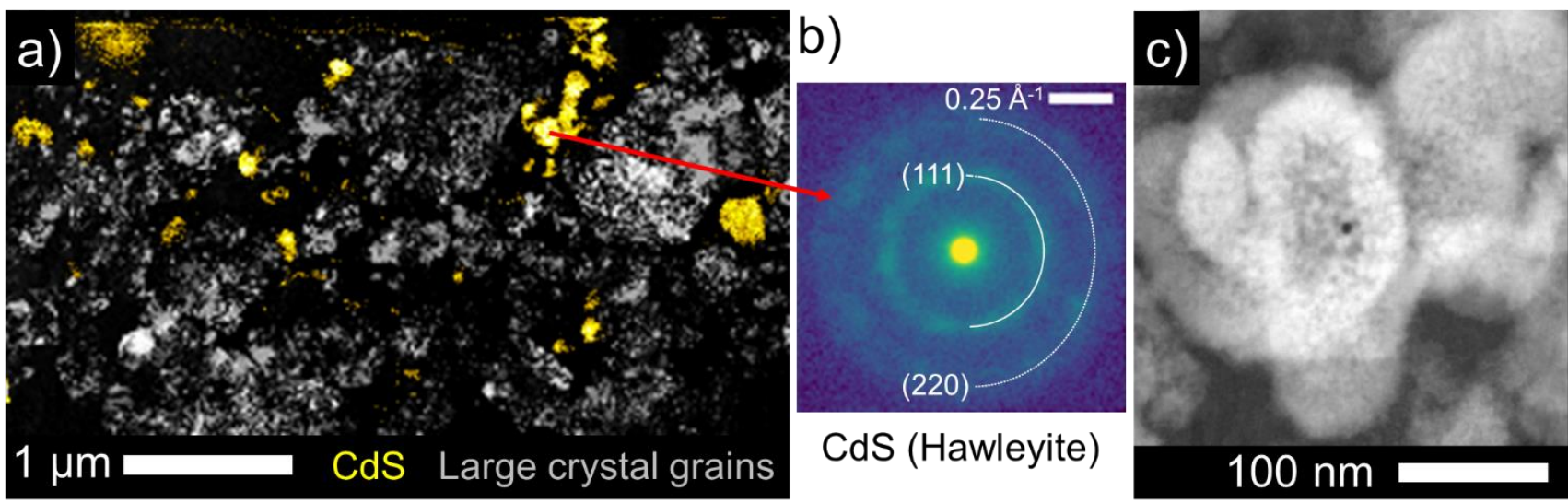

Figure 2. a) Overlay of sulfur EELS signal (CdS) with map showing large (>10 nm) crystal grains. $\mathrm{CdS}$ particles are almost all located in regions with no large grains. b) Diffraction pattern from $\mathrm{CdS}$ showing polycrystalline rings, which index to the (111) and (220) spacings of Hawleyite. c) Annular dark field (ADF) STEM of a typical CdS region comprised of many sub-10 nm CdS nanoparticles. 